Pacific Journal of Mathematic

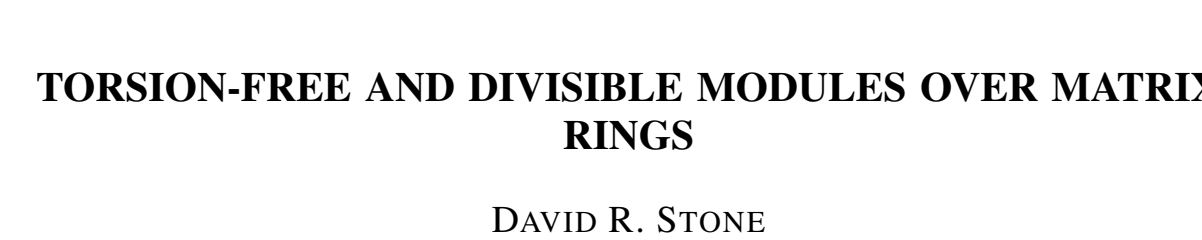




\title{
TORSION-FREE AND DIVISIBLE MODULES OVER MATRIX RINGS
}

\author{
DAVID R. STONE
}

\begin{abstract}
A short exact sequence $0 \rightarrow K \rightarrow F \rightarrow E \rightarrow 0$ of left modules over a ring $A$ is 1-pure if $a K=K \cap a F$ for all $a \in A$, and pure if for any right $A$-module $M$, the map $M \otimes K \rightarrow M \otimes F$ is injective. A module $E$ is torsion free (Hattori) if its presence on the right forces 1-purity, and flat if it forces purity. Similarly, we have on the left the notions of divisibility (Hattori) and absolute purity. Considering the functor $E \rightarrow E^{n}$ taking $A$-modules to modules over the matrix ring $M_{n}(A)$, a sequence is called $n$-pure if its image under this functor is 1-pure; $n$-torsion-free and $n$-divisible modules are similarly defined. It is shown that purity, flatness, and absolute purity, respectively, are equivalent to the requirement that $n$-purity, $n$-torsion-freeness, and $n$-divisibility should hold for all $n$. $n$-divisibility and absolute purity are preserved under direct sums, products and certain inductive limits; $n$-torsion-freeness and flatness under direct sums and inductive limits, but not products. A condition is given guaranteeing that products of at most a given cardinality preserve $n$-torsion-freeness. It is shown that if every left ideal of $A$ is generated by at most $n$ elements, then $n$-torsion-freeness is equivalent to flatness. The behavior of these properties under localization is studied, and it is shown that if $A$ is locally a domain then the two notions of purity agree if and only if $\mathbf{w} \cdot \operatorname{gl}$. $\operatorname{dim} .(A) \leqq 1$.
\end{abstract}

$A$ will always denote a ring with identity; all modules will be unitary and left modules unless otherwise stated. If no confusion can arise $F \otimes E$ will mean $F \bigotimes_{A} E$; similarly for $\operatorname{Hom}(F, E)$, $\operatorname{Tor}_{m}(F, E)$, and $\operatorname{Ext}^{m}(F, E)$.

1. Matrices. For a positive integer $n$, let $M_{n}(A)$ denote the ring of $n \times n$ matrices over $A$ (we shall sometimes use $B=M_{n}(A)$ for convenience of notation) and $M_{n}(E)$ the left $M_{n}(A)$-module of $n \times n$ matrices over $E$, where scalar multiplication looks like usual matrix multiplication. Let $e_{i j} \in M_{n}(A)$ be the matrix having 1 in the $(i, j)$ position and zeros elsewhere.

When considering $E^{n}$ as a left $M_{n}(A)$-module, it is convenient to think of the elements as "column vectors", so we will denote an $n$ tuple of $E^{n}$ as $\left(x_{1}, x_{2}, \cdots, x_{n}\right)^{\prime}$, the prime denoting transpose. Note that $M_{n}(E)$ is a direct sum (as $M_{n}(A)$-modules) of $n$ copies of $E^{n}$. 
The well-known theorem of Morita states that the category of left $A$-modules is category isomorphic to the category of left $M_{n}(A)$ modules by the (exact) functcr $E \rightarrow E^{n}$. (The inverse functor is $M \rightarrow e_{11} M$ for $M$ a left $M_{n}(A)$-module. The $A$-modules $e_{i i} M, i=1, \cdots n$, are all isomorphic and $M \cong\left(e_{11} M\right)^{n}$ by the map $x \rightarrow\left(e_{11}\left(e_{11} x\right), e_{11}\left(e_{12} x\right)\right.$, $\left.\cdots, e_{11}\left(e_{1 n} x\right)\right)^{\prime}$. Also for $E$ a left $A$-module, $E \cong \epsilon_{11} E^{n}$.) The following result is thus obvious.

Proposition 1.1. $E$ is an injective left $A$-module if and only if $E^{n}$ is an injective left $M_{n}(A)$-module (if and only if $M_{n}(E)$ is an injective left $M_{n}(A)$-module).

Proposition 1.2. For $E$ a right $A$-module and $F$ a left $A$ module, $E^{n} \bigotimes_{B} F^{n} \cong E \bigotimes_{A} F$ (as groups). In the situation $\left({ }_{D} E_{A},{ }_{A} F_{C}\right)$ this is an isomorphism of $\left(M_{n}(D), M_{n}(C)\right)$-bimodules.

Proof. Note that $E^{n} \bigotimes_{B} F^{n}$ is generated by the elements of the form $\left(0, \ldots, 0, x_{i}, 0, \cdots\right) \otimes\left(0, \cdots, y_{j}, 0, \cdots 0\right)^{\prime}$ for $x_{i} \in E, y_{j} \in F$, and $x_{i}$ appearing as the $i^{\text {th }}$ coordinate, $y_{j}$ as the $j^{\text {th }}$. But

$$
\begin{aligned}
\left(0, \cdots, x_{i}, \cdots, 0\right) & \otimes\left(0, \cdots, y_{j}, \cdots, 0\right)^{\prime} \\
& =\left(0, \cdots, x_{i}, \cdots, 0\right) \otimes e_{j i}\left(y_{j}, 0, \cdots, 0\right)^{\prime} \\
& =\left(0, \cdots, x_{i}, \cdots, 0\right) e_{j i} \otimes\left(y_{j}, 0, \ldots, 0\right)^{\prime} \\
& =\left(\delta_{i j} x_{i}, 0,0, \cdots, 0\right) \otimes\left(y_{j}, 0, \cdots, 0\right)^{\prime},
\end{aligned}
$$

where $\delta_{i j}$ is the Kronecker delta. Thus the elements $(x, 0, \ldots, 0) \otimes$ $(y, 0, \cdots, 0)^{\prime}$, for $x \in E, y \in F$, generate $E^{n} \bigotimes_{B} F^{n}$.

There is a group homomorphism $E^{n} \bigotimes_{B} F^{n} \rightarrow E \otimes_{A} F$ such that $\left(x_{1}, x_{2}, \ldots, x_{n}\right) \otimes\left(y_{1}, y_{2}, \cdots, y_{n}\right)^{\prime} \rightarrow \sum_{i=1}^{n} x_{i} \otimes y_{i}$. It is clear from the above that the inverse of this is the map $E \bigotimes_{A} F \rightarrow E^{n} \bigotimes_{B} F^{n}$ such that $x \otimes y \rightarrow(x, 0, \cdots, 0) \otimes(y, 0, \cdots, 0)^{\prime}$.

The final assertion follows from the form of the isomorphism.

Since Tor is categorically defined, we have

CoROLlaRY 1.3. $\operatorname{Tor}_{m}^{B}\left(E^{n}, F^{n}\right) \cong \operatorname{Tor}_{m}^{A}\left(E, F^{\prime}\right)$.

Corollary 1.4. w.gl. $\operatorname{dim} A=$ w.gl. $\operatorname{dim} M_{n}(A)$.

2. Purity. We shall call a short exact sequence

$$
0 \longrightarrow K \longrightarrow F \longrightarrow E \longrightarrow 0
$$

of left $A$-modules 1-pure if $a F \cap K=a K$ for every $a \in A$; that is, if and only if $A / a A \otimes K \rightarrow A / a A \otimes F$ is an injection for every $a \in A$. Also (*) is pure (or $K$ is a pure submodule of $F$ ) if for every right 
$A$-module $G$, the map $G \otimes K \rightarrow G \otimes F$ is an injection. (This latter definition is due to P.M. Cohn [5]. In [19], Warfield uses " $R D$ pure" for our "1-pure".) Every pure exact sequence is clearly 1-pure. Note that it is sufficient to consider only finitely generated (presented) modules $G$ in the definition of pure, since any module is a direct limit of finitely generated (presented) modules and tensor products commute with direct limits.

For a positive integer $m$, we say that $(*)$ is $m$-pure if $0 \rightarrow K^{m}$ $\rightarrow F^{m} \rightarrow E^{m} \rightarrow 0$ is a 1 -pure extension of $M_{m}(A)$-modules. Note that this is equivalent to requiring 1-purity of $0 \rightarrow M_{m}(K) \rightarrow M_{m}(F) \rightarrow$ $M_{m}(E) \rightarrow 0$.

Proposition 2.1. If $n \geqq m$ and (*) is n-pure, then (*) is m-pure.

Proof. For $a \in M_{m}(A)$, show that $a F^{m} \cap K^{m}=a K^{m}$ by imbedding $m$-tuples in $n$-tuples, using the $n$-purity of $(*)$, and then extracting the needed $m$-tuples.

THEOREM 2.2. (*) is pure if and only if $(*)$ is $n$-pure for all $n \geqq 1$.

Proof. First suppose that $(*)$ is pure and let $n$ be a positive integer, $G$ a right $M_{n}(A)$-module. Then $G \cong M^{n}$ for a right $A$-module $M$. Since $(*)$ is pure, the sequence $0 \rightarrow M \otimes K \rightarrow M \otimes F$ is exact, so by Proposition 1.2, $0 \rightarrow M^{n} \bigotimes_{B} K^{n} \rightarrow M^{n} \bigotimes_{B} F^{n}$ is exact, or equivalently, $0 \rightarrow G \otimes_{B} K^{n} \rightarrow G \otimes_{B} F^{n}$ is exact. Thus $0 \rightarrow K^{n} \rightarrow F^{n} \rightarrow E^{n} \rightarrow 0$ is pure, so 1 -pure, so $(*)$ is $n$-pure.

Conversely, we must show that $G \otimes K \rightarrow G \otimes F$ is an injection for any finitely presented right $A$-module $G$. However, for such a $G$ there exist free right $A$-modules $F_{2}$ (on $n$ generators) and $F_{1}$ (on $m$ generators), with $n>m$, such that $F_{2} \stackrel{\tau}{\rightarrow} F_{1} \rightarrow G \rightarrow 0$ is exact. Let $b$ be the $m \times n$ matrix associated with the homomorphism $\tau$, and let $M_{m, n}(A)$ be the right $M_{n}(A)$-module of $m \times n$ matrices. Now $F_{2}^{n} \stackrel{\tau^{n}}{\rightarrow} F_{1}^{n} \rightarrow G^{n} \rightarrow 0$ is exact, $F_{2}^{n} \cong M_{n}(A), F_{1}^{n} \cong M_{m, n}(A)$ (as right $M_{n}(A)$-modules) and the image of $\tau^{n}$ is carried under the isomorphism to $b M_{n}(A)$, so $G^{n} \simeq M_{m, n}(A) / b M_{n}(A)$. Let $\iota: M_{m, n}(A) \rightarrow M_{n}(A)$ be the $M_{n}(A)$-linear injection taking an $m \times n$ matrix $a$ to the $n \times n$ matrix $c(a)$ obtained by tacking on $n-m$ rows of zeros. Noting that the $M_{n}(A)$-linear map $\alpha: M_{m, n}(A) / b M_{n}(A) \rightarrow M_{n}(A) / c(b) M_{n}(A)$ by $\alpha\left(a+b M_{n}(A)\right)=\iota(a)+\iota(b) M_{n}(A)$ has an abvious left inverse, we see that the vertical maps in the following commutative square are injections:

$$
\begin{gathered}
M_{m, n}(A) / b M_{n}(A) \otimes K^{n} \longrightarrow M_{m, n}(A) / b M_{n}(A) \otimes F^{n} \\
\alpha \otimes 1 \downarrow 1 \downarrow \\
M_{n}(A) / \iota(b) M_{n}(A) \otimes K^{n} \longrightarrow M_{n}(A) / \iota(b) M_{n}(A) \otimes F^{n}
\end{gathered}
$$


The $n$-purity of $(*)$ yields the injectivity of the lower map and thus of the upper map. Hence $G^{n} \otimes K^{n} \rightarrow G^{n} \otimes F^{n}$ is an injection, so $G \otimes K \rightarrow G \otimes F$ is an injection.

COROLlaRY 2.3. (Cohn's criterion for purity.) $K$ is pure in $F$ if and only if whenever $a_{i j} \in A, y_{j} \in F, x_{i} \in K(i, j=1, \cdots, n)$ and $x_{i}=\sum_{j=1}^{n} a_{i j} y_{j}$ for each $i$, then there are $z_{j} \in K(j=1, \cdots, n)$ such that $x_{i}=\sum_{j=1}^{n} a_{i j} z_{j}$ for each $i$.

Note that in order to prove $(*)$ pure it is sufficient, by Proposition 2.1 and Theorem 2.2, to prove that $(*)$ is $n$-pure for $n \in J$, where $J$ is a cofinal subset of the positive integers.

Corollary 2.4. For a positive integer $m, K$ is pure in $F$ if and only if $K^{m}$ is pure in $F^{m}$; i.e., $(*)$ is pure if and only if $0 \rightarrow K^{m} \rightarrow F^{m} \rightarrow E^{m} \rightarrow 0$ is pure.

Proof. The necessity was shown in the above proof. For the sufficiency, note that $M_{n}\left(M_{m}(A)\right) \cong M_{m n}(A)$ (as rings) and $\left(E^{m}\right)^{n} \cong E^{m n}$ as modules over this ring. Now apply the theorem and the above remark.

That is, purity of an $A$-submodule is equivalent to purity of the equivalent $M_{n}(A)$-submodule. We shall see that the same cannot be said of 1-purity.

This corollary also says that if $0 \rightarrow K^{m} \rightarrow F^{m} \rightarrow E^{m} \rightarrow 0$ is pure for some $m \geqq 1$, it is pure for every $m \geqq 1$.

For a group $G$, let $M(G)$ denote the set of infinite matrices over $G$ which are eventually zero except for a constant down the diagonal. Then $M(A)$ is a ring and $M(E)$ an $M(A)$-module.

For $N$ the set of positive integers, let $E^{(N)}$ be the left $M(A)$ module of families $\left(x_{n}\right)_{n \in N}$ from $E$ with finite support. Note that if $a \in M(A)$, there is an $n \in N$, a matrix $\left(a_{i j}\right) \in M_{n}(A)$, and an element $c \in A$ such that

$$
a=\left(\begin{array}{c:c}
a_{i j} & 0 \\
\hdashline 0 & C
\end{array}\right)
$$

where $C$ is the infinite diagonal matrix $C=\operatorname{diag}(c, c, c, \cdots)$.

COROLlary 2.5. (*) is pure if and only if $0 \rightarrow M(K) \rightarrow M(F)$ $\rightarrow M(E) \rightarrow 0$ (respectively $0 \rightarrow K^{(N)} \rightarrow F^{(N)} \rightarrow E^{(N)} \rightarrow 0$ ) is a 1-pure extension of $M(A)$-modules.

A left $A$-module $P$ is said to be $R D$-projective (pure-projective) if for any 1-pure (pure) exact sequence (*) of left $A$-modules, the induced map $\operatorname{Hom}(P, F) \rightarrow \operatorname{Hom}(P, E)$ is a surjection. Every $R D$ - 
projective module is pure-projective. Warfield has shown in [19] (where he defines the notion) that a module is $R D$-projective if and only if it is a direct summand of a direct sum of cyclically presented modules (after Kaplansky [14], we shall call an $A$-module cyclically presented if it is of the form $A / A a$ for some $a \in A$ ), and that a module is pure-projective if and only if it is a summand of a direct sum of finitely presented modules. In particular, any finitely presented module is pure-projective. The following result was suggested by Warfield.

Proposition 2.6. For a ring $A$, the following conditions are equivalent.

(a) Every 1-pure extension (*) of left A-modules is pure.

(b) Every finitely presented left A-module is a summand of a direct sum of cyclically presented modules.

(c) Every finitely presented left A-module is RD-projective.

(d) Every pure-projective left A-module is RD-projective.

(a') Every 1-pure extension (*) of right A-modules is pure.

$\left(b^{\prime}\right)$ Every finitely presented right A-module is a summand of a direct sum of cyclically presented modules.

$\left(\mathrm{c}^{\prime}\right)$ Every finitely presented right $A$-module is $R D$-projective.

(d') Every pure-projective right A-module is RD-projective.

Proof. By the remarks above, $(b) \Leftrightarrow(c),(d) \Rightarrow(c),\left(b^{\prime}\right) \Leftrightarrow\left(c^{\prime}\right)$ and $\left(d^{\prime}\right) \Rightarrow\left(c^{\prime}\right)$. Clearly $(a) \Rightarrow(d)$ and $\left(a^{\prime}\right) \Rightarrow\left(d^{\prime}\right)$.

To prove that $\left(b^{\prime}\right) \Rightarrow(a)$, we must show that if $(*)$ is a 1-pure extension of left $A$-modules and $H$ a finitely presented right $A$-module, then $0 \rightarrow H \otimes K \rightarrow H \otimes F$ is exact. But by (b'), $H \oplus L=G=\bigoplus_{i \in I} A / a_{i} A$, for $a_{i} \in A, I$ some index set. Then $0 \rightarrow A / a_{i} A \otimes K \rightarrow A / a_{i} A \otimes F$ is exact for each $i \in I$ by hypothesis, so $0 \rightarrow \oplus\left(A / a_{i} A \otimes K\right) \rightarrow \oplus\left(A / a_{i} A\right.$ $\otimes F)$, or $0 \rightarrow G \otimes K \rightarrow G \otimes F$, is exact. That is, $0 \rightarrow(H \oplus L) \otimes K$ $\rightarrow(H \oplus L) \otimes F$ is exact, so the desired conclusion follows. Similarly, (b) $\Rightarrow\left(a^{\prime}\right)$.

For example, Warfield has shown in [19] that if $A$ is a valuation ring then condition (b) is satisfied. This leads us to localization.

If $S$ is a multiplicatively closed subset of the center of $A$ (with $0 \notin S, 1 \in S$ ), let $S^{-1} A$ denote the ring of fractions of $A$ having elements of $S$ as denominators and $S^{-1} E$ the $S^{-1} A$-module of fractions of $E$. (In particular, if $S$ is the complement of a maximal ideal $\mathscr{C}$ of $A$ we employ the usual notation: $S^{-1} A=A_{\mathscr{N}}$ and $S^{-1} E=E_{\mathscr{N}}$.) Since the center of $A$ is also the center of $M_{n}(A)$, we can form the ring of fractions $S^{-1} M_{n}(A)$. It is easy to check that the map $\left(a_{i j}\right) / s \rightarrow$ $\left(a_{i j} / s\right)$ from $S^{-1} M_{n}(A)$ to $M_{n}\left(S^{-1} A\right)$ is a ring isomorphism. Then $S^{-1} E^{n}$ $\simeq\left(S^{-1} E\right)^{n}$ as modules over this ring. 
Lemma 2.7. If $S$ is a multiplicative subset of the center of $A$ and $(*)$ is an n-pure extension of left $A$-modules, then $0 \rightarrow S^{-1} K \rightarrow$ $S^{-1} F \rightarrow S^{-1} E \rightarrow 0$ is an n-pure extension of left $S^{-1} A$-modules.

Proof. This follows immediately from the definition for $n=1$; then use the above remarks.

Proposition 2.8. Let $A$ be a commutative ring. Then $(*)$ is 1-pure (pure) if and only if $0 \rightarrow K_{\mathscr{N}} \rightarrow F_{\mathscr{N}} \rightarrow E_{\mathscr{N}} \rightarrow 0$ is a 1-pure (pure) extension of $A_{\mathscr{M}}$-modules for cvery maximal idcal $\mathscr{t}$ of $A$.

Proof. Let $\mathscr{C}$ be a maximal ideal. If $(*)$ is 1 -pure, then $0 \rightarrow$ $K_{\mathscr{A}} \rightarrow E_{\mathscr{L}} \rightarrow F_{\mathscr{M}} \rightarrow 0$ is 1-pure by the lemma, with $S=A-\mathscr{M}$. And if $(*)$ is pure, then it is $n$-pure for every $n \geqq 1$, so again by the lemma, $0 \rightarrow K_{\mathscr{M}} \rightarrow F_{\mathscr{N}} \rightarrow E_{\mathscr{M}} \rightarrow 0$ is $n$-pure for every $n \geqq 1$ and thus pure by Theorem 2.2.

The proofs of sufficiency follow easily, since $G \otimes K \rightarrow G \otimes F$ is an injection if and only if $(G \otimes K)_{\mathscr{N}} \rightarrow(G \otimes F)_{\mathscr{N}}$ is an injection for every and since $S^{-1}$ commutes with tensor products.

Hence if $A$ is commutative, every 1-pure extension of $A$-modules is pure if and only if for every maximal ideal $\mathscr{N}$, every 1-pure extension of $A_{\mathscr{M}}$-modules is pure. (For the demonstration of this, recall that if $C$ is an $A_{\mathscr{M}}$-module then $C$ can also be considered an $A$-module and $C_{\mathscr{N}} \cong C$ as $A_{\mathscr{M}}$-modules.) Thus when investigating the equivalence of 1-pure and pure, one may assume that $A$ is local.

Corollary 2.9. If $A$ is commutative and w.gl. $\operatorname{dim} .(A) \leqq 1$, then any 1-pure extension of $A$-modulss is pure.

Proof. Endo has shown in [7] that w. gl. $\operatorname{dim} .(A) \leqq 1$ if and only if each $A_{\mathscr{N}}$ is a valuation ring.

Warfield has shown in [19] that the converse is true if $A$ is a commutative integral domain.

3. Torsion-free and flat modules. For $a \in A$, let ${ }_{r}(a)$ denote the right annihilator ideal of $a$ in $A$. After Hattori [11] we say that a left $A$-module $E$ is torsion-free if whenever $a \in A, x \in E$ and $a x=0$, then $x \in{ }_{r}(a) E$. That is, $E$ is torsion-free if whenever $a x=0$ we can write $x=\sum_{i=i}^{n} b_{i} y_{i}$ with each $y_{i} \in E, b_{i} \in A$, and $a b_{i}=0$. This definition agrees with the usual one if $A$ is an integral domain, for in this case ${ }_{r}(a)=0$. Hattori obtained the following characterization [11].

Proposition 3.1. $E$ is torsion-free if and only if $\operatorname{Tor}_{1}(A / a A, E)$ $=0$ for every $a \in A$. 
Thus the direct sum of a family of $A$-modules is torsion-free if and only if each is torsion-free. Any free module, and even any flat module, is torsion-free. Note that $E$ is a torsion-free $A$-module if and only if $E^{n}$ is a torsion-free $A$-module. We use the preceding proposition to extend another Hattori result.

Proposition 3.2. For a left A-module $E$, the following are equivalent.

(a) $E$ is torsion-free.

(b) There exists a 1-pure extension (*) of left A-modules with $F$ torsion-free.

(c) Every exact sequence (*) is 1-pure.

Proof. (c) $\Rightarrow$ (b) since there always exists an exact sequence $(*)$ with $F$ free. Consider the above proposition and the exact sequence $\operatorname{Tor}_{1}(A / a A, F) \rightarrow \operatorname{Tor}_{1}(A / a A, E) \rightarrow A / a A \otimes K \rightarrow A / a A \otimes F$ for the implications $(\mathrm{a}) \Rightarrow$ (c) and $(\mathrm{b}) \Rightarrow(\mathrm{a})$.

EXAMPLE. If $n$ and $m$ are positive integers, then $E=Z / m Z$ can be made into a module over $Z / m n Z$. Then $E$ is torsion-free if and only if $m$ and $n$ are relatively prime.

We shall call $E$ n-torsion-free if $E^{n}$ is a torsion-free $M_{n}(A)$ module. Note that a free module is $n$-torsion-free for all $n \geqq 1$ and that the direct sum of a family of $A$-modules is $n$-torsion-free if and only if each is $n$-torsion-free. We obtain an easily-proven result analogous to the one above.

Proposition 3.3. For a left A-module $E$, the following are equivalent.

(a) $E$ is n-torsion-free.

(b) There exists an n-pure extension (*) with $F$ n-torsion-free.

(c) Every exact sequence $0 \rightarrow K^{\prime} \rightarrow F^{\prime} \rightarrow E^{n} \rightarrow 0$ of left $M_{n}(A)$ modules is 1-pure.

(d) Every exact sequence (*) is n-pure.

Recalling that if $n \geqq m$ then an $n$-pure extension is $m$-pure, we have

COROLLARY 3.4. If $n \geqq m$, an $n$-torsion-free module is $m$ torsion-free.

Another similar well-known result follows from the definitions of flat and pure. 
Proposition 3.5. For a left A-module $E$, the following are equivalent.

(a) $E$ is flat.

(b) There exists a pure extension (*) with $F$ flat.

(c) Every exact sequence $(*)$ is pure.

Corollary 3.6. $E$ is a flat A-module if and only if $E^{n}$ is a flat $M_{n}(A)$-module.

Proof. Choose $(*)$ exact with $F$ free and recall that $(*)$ is pure if and only if $0 \rightarrow K^{n} \rightarrow F^{n} \rightarrow E^{n} \rightarrow 0$ is pure.

CoRollary 3.7. If $F$ is flat and $K$ a submodule, then $F / K$ is flat if and only if $K$ is pure in $F$.

Combining the above proposition, Theorem 2.2, and Proposition 3.3, we have

Theorem 3.8. A module is flat if and only if it is n-torsionfree for all $n \geqq 1$.

Restating the definition in terms of elements, we see that $E$ is $n$-torsion-free if and only if the following condition is satisfied :

If $x_{j} \in E$ and $a_{i j} \in A(i=1, \ldots, n ; j=1, \cdots, n)$ are such that $\sum_{j=1}^{n} a_{i j} x_{j}=0$ for each $i$, then there are $y_{u} \in E, b_{j u} \in A(j=1 \cdots, n$; $u=1, \ldots, m)$ such that $x_{j}=\sum_{u=1}^{m} b_{j u} y_{u}$ for each $j$ and $\sum_{j=1}^{n} a_{i j} b_{j u}=0$ for each $i$ and $u$.

Actually we need not require a square scalar matrix; that is, we could let $i=1, \cdots, r$ and $j=1, \cdots, s$ for any $r, s \leqq n$.

Using this and Theorem 3.8, Chase's criterion for flatness [4, Proposition 1.2 (c)] is easily derived.

The following proposition is a direct generalization of the fact that over a principal ideal ring every torsion-free module is flat.

Proposition 3.9. If every finitely generated right ideal of $A$ is generated by at most $n$ elements, then any $n$-torsion-free left $A$ module is flat.

Proof. Let $E$ be an $n$-torsion-free left $A$-module. To show $E$ flat, it suffices to show $\mathscr{A} \otimes E \rightarrow A \otimes E=E$ is an injection for $\mathscr{A}$ any finitely generated right ideal. By hypothesis, $\mathscr{A}=a_{1} A+a_{2} A$ $+\cdots+a_{n} A$, so if $z \in \mathscr{A} \otimes E$ we can write $z=\sum_{i=1}^{n} a_{i} \otimes x_{i}$, for $x_{i} \in E$. But if $\sum_{i=1}^{n} a_{i} x_{i}=0$ there are elements $y_{j} \in E, a_{i j} \in A(i=1$, 
$\cdots n ; j=1, \cdots m)$ such that $x_{i}=\sum_{j=1}^{m} b_{i j} y_{j}$ for each $i$ and $\sum_{i=1}^{n} a_{i} b_{i j}=0$ for each $j$. Thus

$$
\sum_{i=1}^{n} a_{i} \otimes x_{i}=\sum_{i=1}^{n} \alpha_{i} \otimes\left(\sum_{j=1}^{m} b_{i j} y_{j}\right)=\sum_{j=1}^{m}\left(\sum_{i=1}^{n} a_{i} b_{i j}\right) \otimes y_{j}=0,
$$

so the map is an injection.

COROLLARY 3.10. If every finitely generated right ideal of $A$ is generated by at most $n$ elements, then any torsion-free left $M_{n}(A)$ module is flat.

Proof. If $M$ is a torsion-free left $M_{n}(A)$-module, then $M \cong E^{n}$ for a left $A$-module $E$ which is by definition $n$-torsion-free.

By the proposition, $E$ is a flat $A$-module, so by Corollary 3.6, $E^{n} \cong M$ is a flat $M_{n}(A)$-module.

Considering the "other side" of the tensor product, we have the following similarly proven result.

Proposition 3.11. If a left A-module is generated by no more than $n$ elements and is n-torsion-free, then it is flat.

In particular, any cyclic torsion- free module or any torsion-free principal ideal is flat.

C. U. Jensen [13] has proved the following proposition for the case where $A$ has no zero divisors, but his proof can be easily adapted to our more general situation.

Proposition 3.12. A torsion-free left $A$-module $E$ is flat if and only if for all (finitely generated) right ideals $U$ and $V$ we have $(U \cap V) E=U E \cap V E$.

We would like to have a similar criterion telling when a torsionfree module is $n$-torsion-free, but we have only been able to show that if $E$ is $2 n$-torsion-free then $(U \cap V) E=U E \cap V E$ for $U$ and $V$ right ideals which are generated by at most $n$ elements.

ExAMPLE. Let $A$ be a commutative integral domain with $p$ and $q$ two inequivalent primes such that $\mathscr{A}=A_{p}+A_{q} \neq A$. Then $\mathscr{A}$ is torsion-free but not 2-torsion-free. For letting

$$
a=\left(\begin{array}{cc}
-q & p \\
0 & 0
\end{array}\right) \in M_{2}(A) \text { and } x=\left(\begin{array}{l}
p \\
q
\end{array}\right) \in \mathscr{A}^{2} \text {, then } a x=0 .
$$

The right annihilator of $a$ in $M_{2}(A)$ consists of all matrices of the form $\left(\begin{array}{cc}p b & p c \\ q b & q c\end{array}\right)=\left(\begin{array}{ll}p & 0 \\ q & 0\end{array}\right)\left(\begin{array}{ll}b & c \\ 0 & 0\end{array}\right)$ for $b, c \in A$. It is easy to see that 
$x \notin r(a) \mathscr{A}^{2}$

For example, if $K$ is a commutative field and $A=K[X, Y]-$ the polynomials in two indeterminates with coefficients from $K$-then $X$ and $Y$ are inequivalent primes, so the ideal of polynomials with no constant term is torsion-free but not 2-torsion-free.

ExAMPLE. Let $K$ be a commutative field, $K$ [[X]] the ring of formal power series with coefficients in $K$, and let $A$ be the subring of $K[[X]]$ consisting of all power series without terms of degree 1 (cf. [3], Chapter VIII, Exercise 10). Then $A$ is a local domain having maximal ideal the set of power series of order at least two. This maximal ideal is generated by $X^{2}$ and $X^{3}$, so if it were 2-torsion-free it would be flat, hence free (since $A$ is local). Since it is not free, this gives another example of a torsion-free module which is not 2-torsion-free.

Note that these examples show that we cannot replace "pure" by "1-pure" in Corollary 2.4 .

Unfortunately, we do not yet have an example of a module which is 2-torsion-free but not 3 -torsion-free, so it is conceivable that 2torsion-free may be equivalent to flat.

If $f: A \rightarrow C$ is a homomorphism of rings and $E$ a left $C$-module, then $C$ and $E$ can be made into left $A$-modules. If $C$ is an $n$-torsion free $A$-module and $E$ an $n$-torsion-free $C$-module, then $E$ is an $n$ torsion-free $A$-module. $\quad A s$ is shown by the following example, however, the converse is not true, even for the specialized case where $n=1, C=M_{2}(A)$, and $f: A \rightarrow M_{2}(A)$ is the canonical imbedding.

ExAmple. Let $Z$ be the ring of integers, let $p$ and $q$ be two inequivalent primes of $Z$ and let $\mathscr{A}$ be the principal left ideal of $M_{2}(Z)$ generated by $a=\left(\begin{array}{ll}p & q \\ 0 & 0\end{array}\right)$. Let $E=M_{2}(Z) / \mathscr{A}$. Then $E$ is torsion-free (hence flat) as a $Z$-module but not as an $M_{2}(Z)$-module.

Localization. If $S$ is a multiplicative subset of the center of $A$ and $E$ a torsion-free left $A$-module, then $S^{-1} E$ is a torsion-free left $S^{-1} A$-module. Thus, since $M_{n}(-)$ and $E^{n}$ commute with $S^{-1}$, if $E^{\prime}$ is $n$-torsion-free (flat) so is $S^{-1} E$. We want to show the "converse". First we need some notation and preliminaries.

Let $A$ be commutative, let $B=M_{n}(A), C=S^{-1} A$, and $D=$ $S^{-1} M_{n}(A)$. Recall [2] that if $E$ and $F$ are $A$-modules then $S^{-1}\left(E \otimes_{A} F\right)$ $\cong S^{-1} E \otimes_{C} S^{-1} F$.

Lemma 3.13. If $\mathscr{A}$ is an ideal of $A$, then

$$
S^{-1}\left(\operatorname{Tor}_{1}^{A}(A / \mathscr{A}, E)\right) \cong \operatorname{Tor}_{1}^{C}\left(S^{-1} A / S^{-1} \mathscr{A}, S^{-1} E\right) \text {. }
$$


Proof. We have the exact sequence $0 \rightarrow \mathscr{A} \rightarrow A \rightarrow A / \mathscr{A} \rightarrow 0$ so we obtain exact sequences

$$
0 \longrightarrow \operatorname{Tor}_{1}^{C}\left(S^{-1} A / S^{-1} \mathscr{A}, S^{-1} E\right) \longrightarrow S^{-1} \mathscr{A} \otimes_{c} S^{-1} E \longrightarrow S^{-1} E
$$

and

$$
0 \longrightarrow S^{-1}\left(\operatorname{Tor}_{1}^{A}(A / \mathscr{A}, E)\right) \longrightarrow S^{-1}\left(\mathscr{A} \otimes_{A} E\right) \longrightarrow S^{-1} E
$$

Lemma 3.14. If $F$ is a right $M_{n}(A)$-module, $E$ a left $M_{n}(A)$ module, then $S^{-1}\left(F \bigotimes_{B} E\right) \cong S^{-1} F \bigotimes_{D} S^{-1} E$ as $S^{-1} A$-modules. (Note that on the left we are considering $S \subset A$, and on the right, $S \subset$ $M_{n}(A)$.)

Proof. The $S^{-1} A$-linear map $S^{-1} F \otimes_{D} S^{-1} E \rightarrow S^{-1}\left(F \bigotimes_{B} E\right)$ taking $x / s \otimes y / t \rightarrow x \otimes y / s t$ has as inverse the map $x \otimes y / s \rightarrow 1 / s(x / 1 \otimes y / 1)$.

LEMma 3.15. If $\mathscr{A}$ is a right ideal of $M_{n}(A)$ and $E$ a left $M_{n}(A)$-module, then

$$
S^{-1}\left(\operatorname{Tor}_{1}^{B}\left(M_{n}(A) / \mathscr{A}, E\right)\right) \cong \operatorname{Tor}_{1}^{D}\left(S^{-1} M_{n}(A) / S^{-1} \mathscr{A}, S^{-1} E\right) .
$$

Proof. Similar to the proof of Lemma 3.13, but use Lemma 3.14.

THEOREM 3.16. Let $A$ be a commutative ring and $E$ an $A$-module. Then $E$ is n-torsion-free if and only if $E_{\mathscr{A}}$ is an n-torsion-free $A_{\mathscr{M}}$-module for every maximal ideal $\mathscr{M l}$ of $A$.

Proof. Only the sufficiency requires proof. First let $n=1$. Let $a \in A$, let $\mathscr{C}$ be a maximal ideal of $A$ and $S=A-\mathscr{C}$. Then

$S^{-1}\left(\operatorname{Tor}_{1}^{A}(A / a A, E)\right) \cong \operatorname{Tor}_{1}^{A} \mathscr{C}\left(A_{\mathscr{M}} / a A_{\mathscr{M}}, E_{\mathscr{M}}\right)$ by Lemma 3.13 ; if $E_{\mathscr{N}}$ is assumed torsion-free this last group equals zero. Letting $\mathscr{C}$ range through the set of maximal ideals $A$, $\operatorname{Tor}_{1}^{A}(A / a A, E)=0$, so $E$ is torsion-free. For $n>1$ the proof is the same but uses Lemma 3.15 .

Corollary 3.17. $E$ is flat if and only if $E_{\mathcal{M}}$ is flat for every $\mathscr{M}$.

Hence every torsion-free $A$-module is flat if and only if for every maximal ideal $\mathscr{C}$, every torsion-free $A_{\mathscr{K}}$-module is flat.

If $A$ is commutative and w.gl.dim. $A \leqq 1$, we have shown that the notions of 1-pure and pure coincide, so by Proposition 3.2 and Proposition 3.5, the notions of torsion-free and flat also coincide. The converse is not true, for if $A=Z / 4 Z$ then every torsion-free $A$-module is flat, but w. gl. $\operatorname{dim} A=\infty$. (Perhaps torsion-free agrees with flat 
if and only if finitistic w.gl. $\operatorname{dim} . A \leqq 1$.) For an integral domain $A$, 1-pure coincides with pure if and only if torsion-free coincides with flat if and only if w. gl. $\operatorname{dim}$. $A \leqq 1$ (i. e., $A$ is Prufer). We have the following slightly stronger result. Recall first that a ring is said to be loft $P P(P F)$ if every principal left ideal is projective (flat).

Proposition 3.18. If $A$ is a commutative PF ring, then w.gl. $\operatorname{dim} A \leqq 1$ if and only if every torsion-free $A$-module is flat.

Proof. We need only show the sufficiency. Fieldhouse has shown [10] that $A$ is $P F$ if and only if $A_{\mathscr{N}}$ is an integral domain for each maximal ideal $\mathscr{M}$. So if torsion-free agrees with flat over $A$, the same is true over $A_{\mathscr{M}}$, which must thus be a Prufer ring. Since a local Prufer domain is a valuation ring, we have w. gl. dim. $\mathrm{A} \leqq 1$.

Rephrasing this result: w.gl.dim. $A \leqq 1$ if and only if $A$ is $P F$ and torsion-free agrees with flat.

Inductive limits. Let $I$ be a directed set and let $\left(A_{i}, \Phi_{j i}\right)$ be an inductive system of rings. For each $i \in I$, let $E_{i}$ be a left $A_{i}$-module such that $\left(E_{i}, f_{j i}\right)$ is an inductive system of modules and such that if $i \leqq j, x_{i} \in E_{i}, a_{i} \in A_{i}$, then $f_{j i}\left(a_{i} x_{i}\right)=\Phi_{j i}\left(a_{i}\right) f_{j i}\left(x_{i}\right)$. Then $E=\lim _{\rightarrow} E_{i}$ can be made into a left module over $A=\lim _{\rightarrow} A_{i}$ (see [1]). Let $f_{i}: E_{i}$ $\rightarrow E$ and $\Phi_{i}: A_{i} \rightarrow A$ denote the canonical maps. Note that if $n \geqq 1$ then $\lim _{\rightarrow} M_{n}\left(A_{i}\right) \cong M_{n}\left(\lim _{\rightarrow} A_{i}\right)$ as rings and $\lim _{\rightarrow} E_{i}^{n} \cong\left(\lim _{\rightarrow} E_{i}\right)^{n}$ as modules over this ring.

THEOREM 3.19. If each $E_{i}$ is an n-torsion-free $A_{i}$-module, then $E$ is an n-torsion-free A-module.

Proof. By the above remark, it suffices to prove the theorem for $n=1$. Let $a \in A$ and let $k \in I$ be such that $\Phi_{k}\left(a_{k}\right)=a$ for some $a_{k} \in A_{k}$. Let $J=\{i \in I \mid i \geqq k\}$. Then $J$ is cofinal in $I$ so inductive limits over $J$ are the same as over $I$. There is a family $\left(a_{i}\right)_{i \in J}$ such that $\Phi_{j i}\left(a_{i}\right)=a_{j}$ if $i \leqq j$ and $\Phi_{i}\left(a_{i}\right)=a$ for every $i \in J$. Then it is easy to show that $a \lim _{\rightarrow} A_{i} \cong \lim _{\rightarrow} a_{i} A_{i}$ as $A$-modules and hence (since tensor products commute with inductive limits)

$\operatorname{Tor}_{1}^{A}(A / a A, E) \cong \lim _{\rightarrow} \operatorname{Tor}_{1}^{A} i\left(A_{i} / a_{i} A_{i}, E_{i}\right)$. Since each $A_{i}$ is assumed to be torsion-free over $A_{i}$, $\operatorname{Tor}_{1}^{A}(A / a A, E)=\lim _{\rightarrow} 0=0$, so $E$ is torsion-free.

An interesting example of this is obtained by fixing $A$ and $E$ and taking the inductive limit over the positive integers: $M_{\omega}(A)=$ $\lim _{\rightarrow} M_{n}(A)$ and $M_{\omega}(E)=\lim _{\rightarrow} E^{n}$, where the maps $M_{m}(A) \rightarrow M_{n}(A)$ and $E^{n} \rightarrow E^{m}$, defined if and only if $m$ is a multiple of $n$, say $m=n p$, 
are the inbeddings $B \rightarrow M_{p}(B)$ by $b \rightarrow \operatorname{diag}(b, b, \cdots, b)$ and $G \rightarrow G^{p}$ by $x \rightarrow(x, x, \cdots, x)^{\prime}$. Note that since $M_{n}$ commutes with $M_{m}$ and inductive limits, it also commutes with $M$. That is,

$M_{n}\left(M_{\omega}(A)\right) \cong M_{\omega}(A)$ and $\left(E^{\omega}\right)^{n} \cong E^{\omega}$.

Proposition 3.20. For a left A-module $E$, the following are equivalent.

(a) $E$ is flat.

(b) $E^{\omega}$ is a torsion-free $M_{\omega}(A)$-module.

(c) $E^{\omega}$ is a flat $M_{\omega}(A)$-module.

Proof. $\quad(a) \Rightarrow(b)$. If $E$ is flat then $E$ is $n$-torsion-free for each $n \geqq 1$ or $E^{n}$ is torsion-free over $M_{n}(A)$. Thus(b) follows from the preceding theorem.

(b) $\Leftrightarrow(c)$. Since $\left(E^{\omega}\right)^{n} \cong E^{\omega}$, if $E^{\omega}$ is torsion-free it is $n$-torsion-free for any $n \geqq 1$, hence flat.

(b) $\Rightarrow$ (a). We show $E$ flat by showing that linear relations in $E$ arise from linear relations in $A$ [4, Proposition 2.3 (b)]. Suppose $a_{i} \in A$, $x_{i} \in E(i=1, \cdots, n)$ and $\sum_{i=1}^{n} a_{i} x_{i}=0$. Let $x=\left(x_{1}, x_{2}, \cdots, x_{n}\right)^{\prime} \in E^{n}$ and let $a \in M_{n}(A)$ be the matrix having $a_{1}, a_{2}, \cdots, a_{n}$ across the first row and zeros elsewhere. Then $a x=0$ in $E^{n}$. Map $a$ to $M_{\omega}(A)$ and $x$ to $E^{\omega}$, use the fact that $E^{\omega}$ is torsion-free and obtain elements from $E$ and $A$ which yield the desired linear relations.

Recall the definitions of $M(A), M(E)$ and $E^{(N)}$. It can be similarly shown that $E$ is flat (over $A$ ) if and only if $M(E)$ (respectively $E^{(N)}$ ) is torsion-free (over $M(A)$ ).

Direct products. Although arbitrary direct sums of torsion-free modules are torsion-free, this is not true of direct products, as is shown by the following example.

ExAMPLE. Let $K$ be a commutative field and $V$ a vector space over $K$ with a countably infinite base. Let $A$ be the ring $K \times V$ with addition componentwise and multiplication defined by $(a, x)$. $(b, y)=(a b, a y+b x)$ for $a, b \in K$ and $x, y \in V$. Note that as an ideal of $A, V$ cannot be finitely generated, and that if $x \in V$ the annihilator of $x$ in $A$ is $V$. Then it is easy to show that the direct product of a countably infinite number of copies of $A$ is not a torsion-free $A$ module.

Hattori has shown [11] that for a ring $A$, arbitrary products of torsion-free left $A$-modules are torsion-free if and only if ${ }_{r}(a)$ is a finitely generated right ideal for every $a \in A$. Our next proposition is a refinement of this result. First however, we make a definition. 
Let $\zeta$ be an infinite cardinal number. A right ideal $\mathscr{A}$ of $A$ is said to have property $(F, \zeta)$ if for any right ideal $V \subset \mathscr{A}$ which is generated by a set of cardinality at most $\zeta$ there is a finitely generated right ideal $C$ such that $V \subset C \subset \mathscr{A}$. Note that $\mathscr{A}$ has property $(F, \zeta)$ for every infinite cardinal $\zeta$ if and only if $\mathscr{A}$ is finitely generated.

Proposition 3.21. Let $\zeta$ be an infinite cardinal and $I$ a set of cardinality $\zeta$; for each $i \in I$ let $A_{i}$ equal $A$ considered as a left module over itself. Then the following are equivalent.

(a) $\prod_{i \in I} A_{i}$ is a torsion-free left A-module.

(b) $\prod_{i \in I} E_{i}$ is a torsion-free left A-module for any family $\left(E_{i}\right)_{i \in I}$ of torsion-free left $A$-modules.

(c) ${ }_{r}$ (a) has property $(F, \zeta)$ for every $a \in A$.

Proof. It is obvious that (b) implies (a), and the proof that (a) implies (c) is straightforward. To show that (c) implies (b), let $\left(E_{i}\right)_{i \in I}$ be a family of torsion-free left $A$-modules and suppose $a \in A$, $\left(x_{i}\right)_{i \in I} \in \Pi E_{i}$ are such that $a\left(x_{i}\right)_{i \in I}=0$. Then for each $i, a x_{i}=0$, so there is an integer $m_{i}$ and elements

$$
b_{u i} \in r(a), y_{u i} \in E_{i}\left(u=1, \cdots, m_{i}\right) \text { such that } x_{i}=\sum_{u=1}^{m_{i}} b_{u i} y_{u i} .
$$

Let $V$ be the right ideal of $A$ generated by the set

$$
\left\{b_{u i} \mid i \in I, u=1, \cdots, m_{i}\right\} \text {. }
$$

which has cardinality at most $\zeta$. Since $V \subset_{r}(\mathrm{a})$, which is assumed to have property $(F, \zeta), V$ must be contained in a finitely generated ideal $C=b_{1} A+\cdots+b_{m} A$, with $C \subset_{\nu}$ (a). Hence there exist $c_{u i j} \in A$ such that $b_{u i}=\sum_{j=1}^{m} b_{j} c_{u i j}$, so $\left(x_{i}\right)_{i \in I}=\sum_{j=1}^{m} b_{j}\left(\sum_{u=1}^{m_{i}} c_{u i j} y_{u i}\right)_{i \in I}$. Since each $b_{j} \in{ }_{r}(a)$, $\Pi E_{i}$ is torsion-free.

A similar result holds for $n$-torsion-free modules: $\zeta$-size products of $n$-torsion-free $A$-modules are $n$-torsion-free if and only if ${ }_{r}$ (a) satisfies property $(F, \zeta)$ for every $a$ in $M_{n}(A)$.

It can also be shown, using a diagonalization argument similar to Hattori's [11, Proposition 8], that arbitrary products of $n$-torsionfree left $A$-modules are $n$-torsion-free if and only if ${ }_{r}(a)$ is a finitely generated right ideal of $M_{n}(A)$ for every $a$ in $M_{n}(A)$.

EXAMPLE. We conclude this section with an example of a ring in which every ${ }_{r}(a)$ has property $(F, \omega)$ but not all ${ }_{r}(a)$ are finitely generated, where $\omega$ is the cardinality of $Z$. Hence over this ring countable products of torsion-free modules are torsion-free.

Let $I$ be an uncountably infinite set and for each $i \in I$ let $K_{i}$ be 
a jommutative field. Let $V$ be the ideal of $\prod_{i \in I} K_{i}$ consisting of families with at most denumerable support. Let $A$ be the ring $Z \times V$, with multiplication

$$
(n, b) \cdot(m, c)=(n m, n c+m b+b c) \text { for } n, m \in Z, b, c \in V,
$$

and

$$
\text { let } E=A / V \text {. }
$$

Now consider the ring $C=A \times E$ with multiplication

$$
(a, c+V) \cdot(b, d+V)=(a b, a d+c b+V) \text { for } a, b, c, d \in A .
$$

$C$ is a commutative ring with identity which satisfies the above claim. Since we have two rings involved, we use the notation ${ }_{r A}(a)$ (respectively ${ }_{r C}(a)$ ) to denote the annihilator ideal of $a$ in $A$ (respectively in $C)$. Note that if $a \in A$ then ${ }_{r A}(a)$ is a principal ideal. It then follows that if $a \in A-V$ or $b \in V$ then ${ }_{r o}(a, b+V)$ is principal, and that if $a \in V$ and $b \in A-V$ then ${ }_{r c}(a, b+V)={ }_{r c}(a, 1+V)=V_{a} \times E$ where $V_{a}={ }_{r A}(a) \cap V$. The ideal $V_{a} \times E$ is not finitely generated but it does satisfy property $(F, \omega)$.

4. Divisible and absolutely pure modules. For $a \in A$, let $l(a)$ denote the left annihilator ideal of $a$ in $A$. In the same paper already referred to [11], Hattori has defined a left $A$-module $K$ to be divisible if whenever $a \in A, x \in E$ and $l(a) x=0$, then $x \in a K$. That is, $K$ is divisible if and only if $a K=\left\{x \in K \mid l(a) \subset \operatorname{Ann}_{A}(x)\right\}$. Hattori has also noted that $K$ is divisible if and only if $\operatorname{Ext}^{1}(A / A a, K)=0$ for every $a \in A$. Thus every injective module is divisible and direct sums and products of divisible modules are divisible. Dual to the results of the previous section, we have the following definitions and propositions.

Proposition 4.1. For a left $A$-module $K$, the following are equivalent.

(a) $K$ is divisible.

(b) Every exact extension (*) is 1-pure.

(c) There exists a 1-pure extension (*) with $F$ divisible.

Let $I(K)$ denote the injective envelope of $K$.

CoROllary 4.2. $K$ is divisible if and only if $0 \rightarrow K \rightarrow I(K) \rightarrow E \rightarrow 0$ is 1-pure.

We shall say that $K$ is $n$-divisible if $K^{n}$ is a divisible $M_{n}(A)$ module. Since $K$ injective implies $K^{n}$ injective, hence divisible, an injective module is $n$-divisible for every $n \geqq 1$. Since 


$$
\bigoplus_{i \in I} K_{i}^{n} \cong\left(\bigoplus_{i \in I} K_{i}\right)^{n} \text { and } \prod_{i \in I} K_{i}^{n} \cong\left(\prod_{i \in I} K_{i}\right)^{n}
$$

as $M_{n}(A)$-modules, $\oplus K_{i}$ (respectively $\Pi K_{i}$ ) is $n$-divisible if and only if each $K_{i}$ is $n$-divisible. Hence $K$ is $n$-divisible if and only if $M_{n}(K)$ is a divisible $M_{n}(A)$-module.

Proposition 4.3. For a left $A$-module $K$, the following are equivalent.

(a) $K$ is n-divisible.

(b) There exists an n-pure extension (*) with $F$-divisible.

(c) Every exact sequence $0 \rightarrow K^{n} \rightarrow F^{\prime} \rightarrow E^{\prime} \rightarrow 0$ of left $M_{n}(A)$ modules is 1-pure.

(d) Every exact sequence (*) is n-pure.

CoRollary 4.4. If $n \geqq m$, an n-divisible module is m-divisible.

Proof. Use Proposition 2.1.

COROLlary 4.5. $K$ is n-divisible if and only if $0 \rightarrow K \rightarrow I(K) \rightarrow E \rightarrow 0$ is n-pure.

Corollary 4.6. A submodule $K$ of an n-divisible module $F$ is $n$-divisible if and only if $(*)$ is n-pure.

After B. H. Maddox [17 and 18] we call $K$ absolutely pure if it is a pure submodule of every module in which it can be imbedded. That is, $K$ is absolutely pure if and only if every extension $(*)$ is pure. Maddox has shown that any injective module is absolutely pure and that any pure submodule of an injective module is absolutely pure. Thus, since $K$ is pure in $I(K)$ if and only if $K^{n}$ is pure in $I(K)^{n}$ (which is injective), we see that $K$ is an absolutely pure $A$-module if and only if $K^{n}$ is an absolutely pure $M_{n}(A)$-module. Note that an absolutely pure module is divisible.

TheOREM 4.7. A left A-module $K$ is absolutely pure if and only if it is $n$-divisible for every $n \geqq 1$.

Proof. If $K$ is absolutely pure, then for every $n \geqq 1, K^{n}$ is absolutely pure, hence divisible.

Conversely, if $K$ is $n$-divisible for every $n \geqq 1$, then $0 \rightarrow K \rightarrow$ $I(K) \rightarrow E \rightarrow 0$ is $n$-pure for every $n \geqq 1$, hence pure (by Theorem 2.2) so $K$ is pure in $I(K)$, or $K$ is absolutely pure.

Corollary 4. 8. For an A-module $K$, the following are equivalent. 
(a) $K$ is absolutely pure.

(b) There exists a pure extension (*) with $F$ absolutely pure.

(c) Every extension (*) is pure.

COROLlaRY 4.9. If $\left(K_{i}\right)_{i \in I}$ is a family of absolutely pure Amodules, then $\Pi_{i \in I} K_{i}$ is absolutely pure.

Proof. Recall that $\Pi K_{i}$ is $n$-divisible if and only if each $K_{i}$ is $n$-divisible.

COROLlary 4.10. $K$ is an absolutely pure A-module if and only if $M(K)$ is a divisible $M(A)$-module.

We have a partial answer to the question of inductive limits.

Proposition 4.11. If $\left(K_{i}, f_{j i}\right)$ is an inductive system of modules over the inductive system of rings $\left(A_{i}, \Phi_{j i}\right)$, if each $f_{j i}$ is an injection and if each $K_{i}$ is a divisible $A_{i}$-module, then $\lim _{\rightarrow} K_{i}$ is divisible over $\lim _{\rightarrow} A_{i}$.

Proof. This follows directly from the definitions.

COROLlaRY 4.12. In the same situation, if each $K_{i}$ is n-divisible, then $\lim _{\rightarrow} K_{i}$ is n-divisible.

Proof. $K^{n}$ and $M_{n}(-)$ commute with inductive limits.

COROLLARY 4.13. If each $K_{i}$ is absolutely pure, then $\lim _{\rightarrow} K_{i}$ is absolutely pure.

Maddox obtained this last result in the case where all $A_{i}=A$. The next proof follows one by Maddox, which followed one by $\mathrm{E}$. Matlis.

Proposition 4.14. For a ring $A$, the following are equivalent. ble.

(a) Every quotient module of a divisible left $A$-module is divisi-

(b) The sum of two divisible submodules of a left A-module is divisible.

Proof. If $K$ and $H$ are divisible left $A$-modules, then so is $K \oplus H$, so $K+H$ is divisible if (a) is assumed. Conversely, assume (b) and let $H$ be a submodule of a divisible module $M$. Let $L$ and $N$ be two copies of $M$ and let $P=L \oplus N$ let $\Delta$ be the diagonal of $P$ and let 
$D=\Delta \cap(H \oplus H)$. With $\Phi: P \rightarrow P / D$ the cannonical surjection it is easy to see that $P / D=\Phi(L)+\Phi(N)$; since $\Phi \mid L$ and $\Phi \mid N$ are injections, $M \cong \Phi(L) \cong \Phi(N)$ so by hypothesis $P / D$ is divisible. But

$$
P / D=((L \oplus 0)+D) / D \oplus \Delta / D,
$$

so $\Delta / D \cong M / H$ is divisible.

(Note that the only property of divisibility used in this proof is that $K \oplus H$ is divisible if and only if $K$ and $H$ are divisible. Hence we can prove the proposition for any property satisfying this condition.)

Hattori has shown in [11] that all quotients of divisible left $A$ modules are divisible if and only if $A$ is left $P P$. So if $M_{n}(A)$ is left $P P$ then quotients of $n$-divisible left $A$-modules are $n$-divisible. Hence if $M_{n}(A)$ is left $P P$ for every $n \geqq 1$ then every quotient module of an absolutely pure left $A$-module is absolutely pure. For example, if $A$ is left semi-hereditary then each $M_{n}(A)$ is left semi-hereditary and thus left $P P$. (Surprisingly, the converse is true; that is, if $M_{n}(A)$ is left $P P$ for every $n \geqq 1$ then $A$ is left semi-hereditary. This can be seen by using [16, Corollary 2.3].) Hence if $A$ is left semi-hereditary, quotients and finite sums of absolutely pure left $A$-modules are absolutely pure, and by one of Maddox' theorems, every left $A$-module has a unique maximal absolutely pure submodule. We do not know whether any of these imply that $A$ is left semi-hereditary.

A classical theorem says that if $A$ is an integral domain then a torsion-free module is injective if and only if it is divisible. We conclude with an example due to Maddox which shows that this result does not hold in our more general setting.

Example. For $i \in N$, the set of positive integers, let $A_{i}=Z / 4 Z$; then let $A=\prod_{i \in N} A_{i}$ and $E=\bigoplus_{i \in N} A_{i} . \quad E$ is an $A$-module which is flat and absolutely pure, hence torsion-free and divisible, but $E$ is not injective.

The author wishes to thank the referee for his comprehensive report and many valuable suggestions.

\section{REFERENCES}

1. N. Bourbaki, Algèbre, Chapter 2, Hermann, Paris, 1962.

2. - Algèbre Commutative, Chapter 1 and Chapter 2, Hermann, Paris, 1961.

3. H. Cartan and S. Eilenberg, Homological Algebra, Princeton University Press, Princeton, 1956.

4. S. U. Chase, Direct products of modules, Trans. Amer. Math. Soc. 97 (1960), 457473. 
5. P. M. Cohn, On the free product of associative rings I, Math. Z. 71 (1959), 380-398.

6. S. Endo, Note on P. P. rings, Nagoya Math. J. 17 (1960), 167-170.

7. - On semi-hereditary rings, J. Math. Soc. Japan 13 (1961), 109-119.

8. - On flat modules over commutative rings, J. Math. Soc. Japan 14 (1962), 284-291.

9. D. J. Fieldhouse, Purity and Flat Covers, Queen's Preprint Series, Queen's University, Kingston, Ontario, Canada, No. 14, 1967.

10. — Pure simple and indecomposable rings, Queen's Preprint Series, Queen's University, Kingston, Ontario, Canada, No. 19, 1967.

11. A. Hattori, A foundation of torsin theory for modules over general rings, Nagoya Math. J. 17 (1960), 147-157.

12. - On Prufer rings, J. Math. Soc. Japan 9 (1957), 381-385.

13. C. U. Jensen, A remark on flat and projective modules, Canad. J. Math. 18 (1966), 943-949.

14. I. Kaplansky, Infinite Abelian Groups (rev. ed.), University of Michigan Press, Ann Arbor, Michigan, 1969.

15. J. Lambek, Lectures on rings and modules, Blaisdell, Waltham, Mass., 1966.

16. L. Levy, Torsion-free and divisible modules over non-integral-domains, Canad. J. Math. 15 (1963), 132-151.

17. B. H. Maddox, Absolutely pure modules, Ph. D. Thesis, University of South Carolina, 1964.

18. — Absolutely pure modules, Proc. Amer, Math. Soc. 18 (1967), 155-158.

19. R. B. Warfield, Jr., Purity and algebraic compactness for modules, Pacific J. Math. 28 (1969), 699-719.

Received September 10, 1968, and in revised form March 4, 1970. This paper is taken from the author's dissertation, which was directed by Dr. Edgar Enochs at the University of South Carolina, where the author was a NASA Trainee.

UNIVERSITY OF KENTUCKY

Georgia Southern College 



\section{PACIFIC JOURNAL OF MATHEMATICS}

\section{EDITORS}

H. SAmelson

Stanford University

Stanford, California 94305

RICHARd PieRCe

University of Washington

Seattle, Washington 98105

\author{
J. DugundJI \\ Department of Mathematics \\ University of Southern California \\ Los Angeles, California 90007 \\ RICHARD ARENS \\ University of California \\ Los Angeles, California 90024
}

ASSOCIATE EDITORS
E. F. BECKENBACH
B. H. NeumanN
F. WOLF
K. YosHIDA

\section{SUPPORTING INSTITUTIONS}

UNIVERSITY OF BRITISH COLUMBIA

CALIFORNIA INSTITUTE OF TECHNOLOGY

UNIVERSITY OF CALIFORNIA

MONTANA STATE UNIVERSITY

UNIVERSITY OF NEVADA

NEW MEXICO STATE UNIVERSITY

OREGON STATE UNIVERSITY

UNIVERSITY OF OREGON

OSAKA UNIVERSITY

UNIVERSITY OF SOUTHERN CALIFORNIA
STANFORD UNIVERSITY

UNIVERSITY OF TOKYO

UNIVERSITY OF UTAH

WASHINGTON STATE UNIVERSITY

UNIVERSITY OF WASHINGTON

AMERICAN MATHEMATICAL SOCIETY CHEVRON RESEARCH CORPORATION TRW SYSTEMS

NAVAL WEAPONS CENTER 


\section{Pacific Journal of Mathematics}

\section{Vol. 35, No. $1 \quad$ September, 1970}

B. D. Arendt and C. J. Stuth, On the structure of commutative periodic semigroups ..................................... 1

B. D. Arendt and C. J. Stuth, On partial homomorphisms of semigroups .... 7

Leonard Asimow, Extensions of continuous affine functions ............ 11

Claude Elias Billigheimer, Regular boundary problems for a five-term recurrence relation ................................... 23

Edwin Ogilvie Buchman and F. A. Valentine, A characterization of the

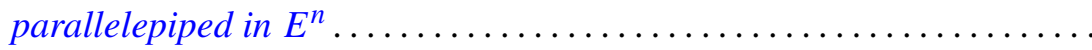
53

Victor P. Camillo, A note on commutative injective rings ............ 59

Larry Jean Cummings, Decomposable symmetric tensors ............. 65

J. E. H. Elliott, On matrices with a restricted number of diagonal values ... 79

Garth Ian Gaudry, Bad behavior and inclusion results for multipliers of type

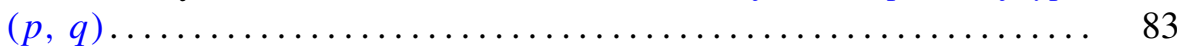

Frances F. Gulick, Derivations and actions .................. 95

Langdon Frank Harris, On subgroups of prime power index . . . . . . . . 117

Jutta Hausen, The hypo residuum of the automorphism group of an abelian

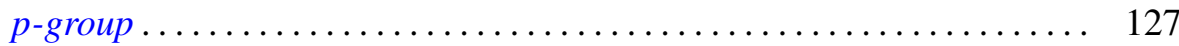

R. Hrycay, Noncontinuous multifuctions . ................... 141

A. Jeanne LaDuke, On a certain generalization of $p$ spaces ............ 155

Marion-Josephine Lim, Rank preservers of skew-symmetric matrices...... 169

John Hathway Lindsey, II, On a six dimensional projective representation of the Hall-Janko group ................................... 175

Roger McCann, Transversally perturbed planar dynamical systems . . . . . 187

Theodore Windle Palmer, Real $C^{*}$-algebras ................... 195

Don David Porter, Symplectic bordism, Stiefel-Whitney numbers, and a

Novikov resolution ....................................... 205

Tilak Raj Prabhakar, On a set of polynomials suggested by Laguerre

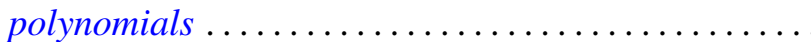

B. L. S. Prakasa Rao, Infinitely divisible characteristic functionals on locally convex topological vector spaces...................

John Robert Reay, Caratheodory theorems in convex product structures ...

Allan M. Sinclair, Eigenvalues in the boundary of the numerical range. .

David R. Stone, Torsion-free and divisible modules over matrix rings ....

William Jennings Wickless, A characterization of the nil radical of a

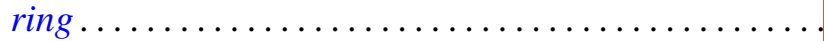

